\title{
Diagnostic Accuracy of Whole-Body Computed Tomography for Incidental Ovarian Tumors in Patients with Prior Breast Cancer
}

\author{
Pei-Ching Huang ${ }^{1,2}$, Ren-Chin Wu ${ }^{3,4} \oplus$, Yu-Hsiang Juan ${ }^{1,2}$, Hui-Yu Ho ${ }^{4,5,6}$, Yung-Chang Lin 4,7,

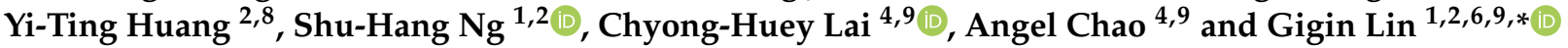

check for updates

Citation: Huang, P.-C.; Wu, R.-C.; Juan, Y.-H.; Ho, H.-Y.; Lin, Y.-C.; Huang, Y.-T.; Ng, S.-H.; Lai, C.-H.; Chao, A.; Lin, G. Diagnostic Accuracy of Whole-Body Computed Tomography for Incidental Ovarian Tumors in Patients with Prior Breast Cancer. Diagnostics 2022, 12, 347.

https: / / doi.org/10.3390/

diagnostics12020347

Academic Editors: Frediano Inzani, Angela Santoro and Gian Franco Zannoni

Received: 22 December 2021

Accepted: 27 January 2022

Published: 29 January 2022

Publisher's Note: MDPI stays neutral with regard to jurisdictional claims in published maps and institutional affiliations.

Copyright: (C) 2022 by the authors. Licensee MDPI, Basel, Switzerland. This article is an open access article distributed under the terms and conditions of the Creative Commons Attribution (CC BY) license (https:// creativecommons.org/licenses/by/ $4.0 /)$.
1 Department of Medical Imaging and Intervention, Chang Gung Memorial Hospital at Linkou, No. 5, Fuxing Str., Guishan Dist., Taoyuan City 33382, Taiwan; spookie@cgmh.org.tw (P.-C.H.); jonat126@yahoo.com.tw (Y.-H.J.); shuhangng@gmail.com (S.-H.N.)

2 Department of Medical Imaging and Radiological Sciences, Chang Gung University, No. 259, Wenhua 1st Rd. Guishan Dist., Taoyuan City 33302, Taiwan; hyt3784@cgmh.org.tw

3 Department of Pathology, Chang Gung Memorial Hospital at Linkou, No. 5, Fuxing Str., Guishan Dist., Taoyuan City 33382, Taiwan; renchin.wu@gmail.com

4 School of Medicine, College of Medicine, Chang Gung University, No. 259, Wenhua 1st Rd., Guishan Dist., Taoyuan City 33302, Taiwan; brook.ho0229@gmail.com (H.-Y.H.); yclinof@adm.cgmh.org.tw (Y.-C.L.); laich46@cgmh.org.tw (C.-H.L.); Drangiechao@gmail.com (A.C.)

5 Department of General Surgery, Chang Gung Memorial Hospital at Linkou, No. 5, Fuxing Str., Guishan Dist., Taoyuan City 33382, Taiwan

6 Clinical Metabolomics Core Lab, Chang Gung Memorial Hospital at Linkou, No. 5, Fuxing Str., Guishan Dist., Taoyuan City 33382, Taiwan

7 Division of Medical Oncology, Department of Internal Medicine, Chang Gung Memorial Hospital at Linkou, No. 5, Fuxing Str., Guishan Dist., Taoyuan City 33382, Taiwan

8 Department of Radiology Oncology, Chang Gung Memorial Hospital at Linkou, No. 5, Fuxing Str., Guishan Dist., Taoyuan City 33382, Taiwan

9 Department of Obstetrics and Gynecology and Gynecologic, Cancer Research Center, Chang Gung Memorial Hospital at Linkou, No. 5, Fuxing Str., Guishan Dist., Taoyuan City 33382, Taiwan

* Correspondence: giginlin@cgmh.org.tw; Tel.: +886-33281200 (ext. 2575)

Abstract: Whole-body computed tomography (WBCT) serves as the first-line imaging modality for breast cancer follow-up. To investigate the imaging characteristics and diagnostic accuracy of WBCT for incidental ovarian tumors in patients with prior breast cancer, we retrospectively reviewed a consecutive cohort of 13,845 patients with breast cancer, of whom 149 had pathologically-proven ovarian lesions. We excluded patients with ovarian diagnosis before breast cancer, CT scan not including ovary, CT-pathology interval $>30$ days, and severe CT artifact. Among our 60 breast cancer patients (median age, 46 years) with pathologically proven ovarian lesions, 49 patients had benign diseases, seven had primary ovarian cancer and four had ovarian metastasis from breast cancer. The histologic types of breast cancer with ovarian metastases included invasive ductal carcinoma, lobular carcinoma and angiosarcoma. Cystic ovarian lesions identified on WBCT during the breast cancer follow-up are more likely to be benign, while solid-cystic lesions are likely to be primary ovarian cancers, and solid lesions may indicate ovarian metastasis. The diagnostic accuracy, sensitivity, specificity, and areas under the receiver operating characteristic curve of WBCT were 98.3\%, 100.0\%, $98.0 \%$, and 0.99 (malignant vs. benign); $90.0 \%, 100.0 \%, 85.7 \%$, and 0.93 (metastasis vs. primary ovarian cancer), respectively. The only false positive solid lesion was a Sertoli-Leydig tumor. In conclusion, WBCT may help diagnose incidental ovarian tumors in patients with prior breast cancers and guide disease management.

Keywords: breast neoplasms; computed tomography; metastasis; ovarian neoplasms

\section{Introduction}

Breast cancer is the most common malignancy in women [1]. Breast cancer can metastasize to bone, lungs, liver and brain, and, although less frequently to the ovaries, either at 
diagnosis or recurrence [2]. The prevalence of ovarian metastases was reported to be $2.4 \%$ among young breast cancer patients [3]. Patients with prior breast cancer are also more likely to develop primary ovarian cancer $[2,4,5]$. Because of a high incidence of breast cancer, its metastasis to the ovaries can constitute substantially of all ovarian neoplasms [2,3]. Distinguishing ovarian metastasis from primary ovarian cancer is important, as the optimal treatments are different and correlated to prognosis. Breast cancer patients with ovarian metastasis should be treated non-surgically, whereas primary ovarian cancers still require optimal cytoreductive surgery as the mainstream treatment [4]. Before obtaining the cancer tissue, the selection of neoadjuvant chemotherapy also depends on this decision: alternative endocrine agents can be considered for the treatment of ovarian metastasis, whereas bevacizumab may be selected for primary ovarian cancer [2]. However, making this decision is also clinically challenging, because of overlapping of the patient demographics, especially when patients with prior breast cancer have an increased risk for developing primary ovarian cancer [6]. Whether any subgroup of breast cancer patients carrying ovarian tumor-like lesions might obviate oophorectomy has yet to be answered because of the increasing prevalence of pre-menopausal or peri-menopausal breast cancer patients [7].

Advanced body imaging can be offered if distant metastasis or disease recurrence is clinically suspected, and this is supported by the American Cancer Society (ACS)/ American Society of Clinical Oncology (ASCO) Breast Cancer Survivorship Care [8] and the National Comprehensive Cancer Network (NCCN) Guidelines [9]. The evaluation of an ovarian lesion years after breast cancer may represent a diagnostic challenge and the possibility of a metastatic lesion should always be considered. Accumulated evidence based on ultrasonography has shown that ovarian metastases derived from breast cancers tend to be solid and vascularized [10-12], and bilaterality has also been reported [4,13]. With technological advancement, whole-body computed tomography (WBCT) is the first-line imaging modality for detecting distant metastasis or recurrence, with the unique capability to scan breasts and ovaries at the same time. In this regard, we conducted a more comprehensive adnexal masses morphological classification, e.g., cystic, cystic-solid and solid lesions, to exam the clinical value of routine WBCT for patients with prior breast cancer.

In this study, we aimed to investigate the imaging characteristics and diagnostic accuracy of WBCT for incidental ovarian tumors in patients with prior breast cancer.

\section{Materials and Methods}

\subsection{Patients}

The institutional review board approved this retrospective study, and informed consent was waived. We retrospectively reviewed a consecutive cohort of 13,845 patients from the breast cancer registry from 2007 to 2018, of whom 149 had pathologically-proven ovarian lesions. Exclusion criteria were (a) diagnosis date of the ovarian pathology before the breast cancer $(n=48)$, colon cancer $(n=2)$ and lung cancer $(n=1)$, and $(b)$ CT scan not including ovary $(n=21)$, CT-pathology interval $>30$ days $(n=15)$, and severe CT artifact $(n=2)$. During data curation, ovarian primary with breast metastasis $(n=3)$ was identified for analyzing the breast-ovarian mutual metastasis. A multidisciplinary team involving imaging experts and gynecologic oncologists discussed the work-up for asymptomatic patients with incidental pelvic lesions on WBCT $(n=40)$ or symptomatic patients with positive findings on transvaginal ultrasound $(n=20)$. Gynecologic oncologists performed pelvic examinations at three to six months and closely monitored tumor markers to decide whether the patients should be admitted for surgery. Transvaginal ( $n=50)$ or transabdominal ultrasound $(n=5)$ was performed before surgery. An ultrasound-guided transvaginal biopsy was not performed in our institution. Serum tumor markers were evaluated, including the levels of carcinoma antigen (CA) 125, CA 15-3, CA19-9, and carcinoembryonic antigen (CEA). 


\section{2. $W B C T$}

WBCT exams were carried out using multi-slice CT systems (Somatom Sensation 4 or 16, Siemens Medical Systems, Erlangen, Germany) if clinically suspected recurrence was indicated during the follow-up. Contiguous axial sections (0.5- $\mathrm{cm}$ thickness) were scanned in a craniocaudal direction between the lower neck and the pelvis, about 60-70 s following intravenous injection of $100 \mathrm{~mL}$ of iodinated contrast medium (Omnipaque 350, Amersham (Cork, Ireland)) or Optiray 350, Mallinckrodt (St. Louis, MO, USA)), at a rate of 2-3 mL/s. Routine coronal reconstruction was done for all CT studies. Two radiologists (G.L. and P.C.H.) reviewed the $\mathrm{CT}$ scans for all patients. Ovarian lesions were classified based on the morphology features (solid, solid-cystic or cystic) and laterality (unilateral or bilateral). The solid lesion was defined by lesions with predominant soft tissue densities with any degree of enhancement on CT. Lesions exhibiting both solid and bilateral patterns were defined as having a combined feature.

\subsection{Histopathologic Analysis}

Surgical histopathology served as the reference standard in this study according to the World Health Organization (WHO) classification 2020, reviewed by a pathologist (R.C.W.) who has 20 years of experience in gynecologic pathology. The clinical information was available for the pathological reference standard. Diagnosis was made primarily based on morphological evidence on the hematoxylin-eosin ( $\mathrm{H} \& \mathrm{E})$-stained tissue sections. An additional immunohistochemical (IHC) analysis was carried out if there was any uncertainty of the diagnosis. The presence of GATA- binding protein 3 (GATA3) was suggestive of metastasis from breast cancer, whereas paired box 8 (PAX8) was suggestive of primary ovarian cancers [14]. The status of estrogen (ER), progesterone (PR) and HER2-neu receptors were used to select an appropriate adjuvant therapy.

\subsection{Statistical Analysis}

Data were analyzed using standard statistical methods on MedCalc for Windows, Version 20.008 (MedCalc Software, Ostend, Belgium). A Mann-Whitney U test was used to compare the continuous parameters, and a Fisher's exact test was used to compare the categorical parameters, respectively. The diagnostic accuracy of WBCT in diagnosis of ovarian lesions was presented with 95\% confidence intervals (CIs). The McNemar test was used to compare the accuracy, sensitivity, and specificity between groups. Areas under the receiver operating characteristic curve (AUCs) were calculated to compare diagnostic performance. A $p$-value $<0.05$ indicated statistical significance in this study.

\section{Results}

\subsection{Patient Characteristics}

A total of 60 patients (median age 46 years, range 24-72) with pathological evidence of the ovary were included in the final analysis, including 49 benign and 11 malignant ovarian lesions. The flowchart of this study is demonstrated in Figure 1. The pathology of breast malignancy comprised invasive ductal carcinoma $(n=52)$, lobular carcinoma $(n=3)$, mucinous adenocarcinoma $(n=2)$, metastatic malignant phyllodes tumor $(n=1)$, papillary carcinoma $(\mathrm{n}=1)$, and angiosarcoma $(\mathrm{n}=1)$. The demographics of the study population are detailed in Table 1. Initial staging of the breast malignancy showed no significant differences between benign and malignant ovary groups in terms of T, N, and M stages. Ovarian pathology proved to be benign in 49 patients, including functional cysts $(n=15)$, endometrioma $(n=10)$, para-ovarian cysts $(n=8)$, cystadenoma $(n=6)$, teratoma $(n=6)$, tubo-ovarian abscess $(\mathrm{n}=3)$, and fibrothecoma $(\mathrm{n}=1)$. Prophylactic oophorectomy was offered for $B R C A$ mutation carriers $(n=2)$ and noncarriers $(n=2)$, with all the pathology results yielded to be functional cysts. The benign lesions were predominantly cystic; even the fibrothecoma appeared as a cystic mass lesion in this cohort. The malignant lesions were solid or solid-cystic. Patients with malignant ovarian lesions had a shorter diagnosis 
interval ( 8 vs. 29 months, $p=0.044)$, and were associated with the M1 stage at diagnosis $(p=0.003)$, as compared with patients with benign ovarian lesions.

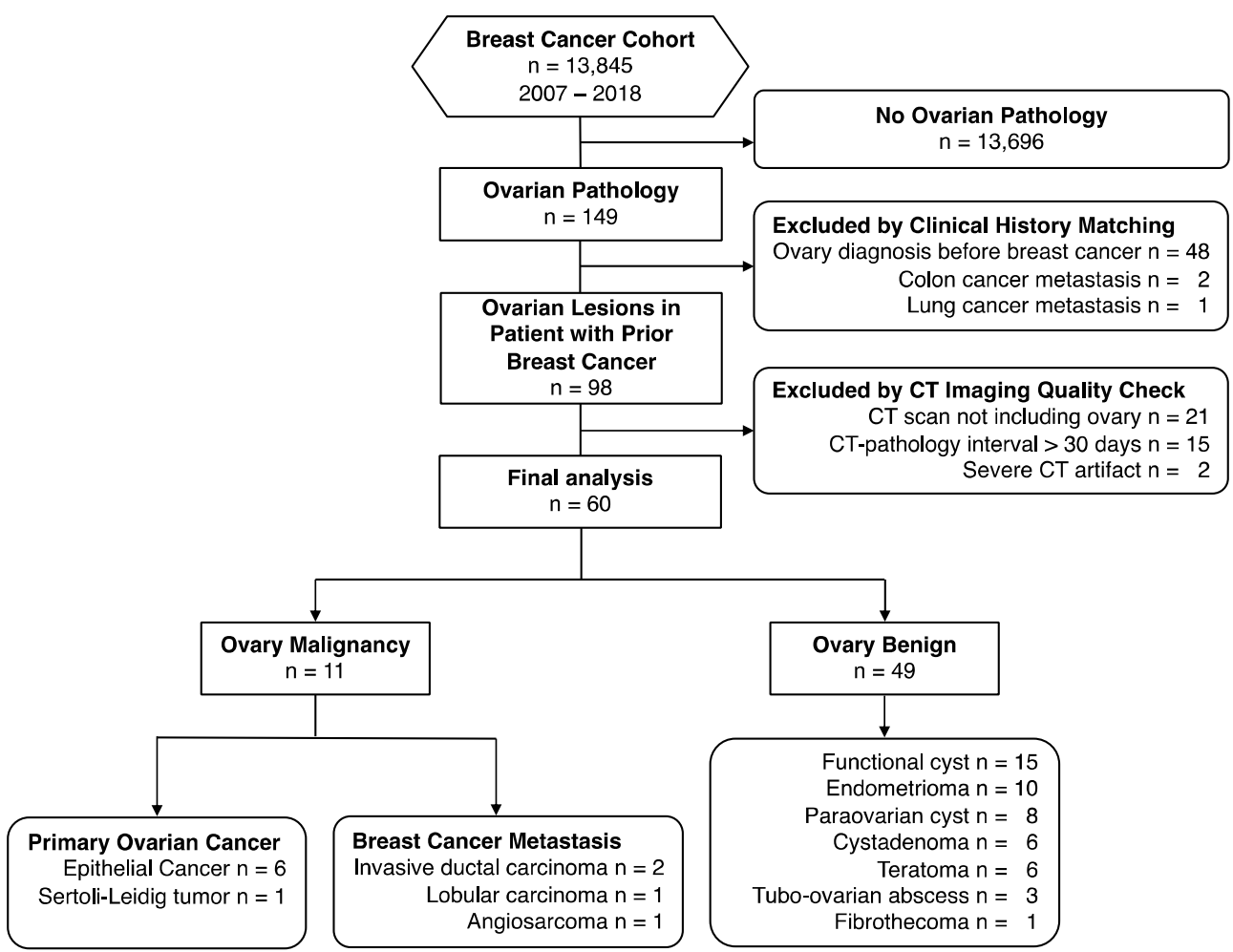

Figure 1. Flow diagram of the study cohort.

Table 1. Demographics of patients with prior breast cancer having incidental ovarian tumors.

\begin{tabular}{|c|c|c|c|c|}
\hline & All & $\begin{array}{c}\text { Ovary } \\
\text { Malignancy }\end{array}$ & Ovary Benign & $p$-Value \\
\hline & $(n=60)$ & $(\mathrm{n}=11)$ & $(n=49)$ & \\
\hline Age, median (year) & $46(24,72)$ & $51(32,60)$ & $45(24,72)$ & 0.203 \\
\hline Breast pathology & & & & 0.390 \\
\hline Invasive ductal carcinoma & 52 & $9(81.8 \%)$ & $43(87.8 \%)$ & \\
\hline Lobular carcinoma & 3 & $1(9.1 \%)$ & $2(4.1 \%)$ & \\
\hline Mucinous adenocarcinoma & 2 & $0(0.0 \%)$ & $2(4.1 \%)$ & \\
\hline Malignant phyllodes tumor & 1 & $0(0.0 \%)$ & $1(2.0 \%)$ & \\
\hline Papillary carcinoma & 1 & $0(0.0 \%)$ & $1(2.0 \%)$ & \\
\hline Angiosarcoma & 1 & $1(9.1 \%)$ & $0(0.0 \%)$ & \\
\hline Morphology & & & & 0.000 \\
\hline Solid or solid-cystic & 11 & $11(100.0 \%)$ & $1(2.0 \%)$ & \\
\hline Cystic & 49 & $0(0.0 \%)$ & $48(98.0 \%)$ & \\
\hline Laterality & & & & 0.154 \\
\hline Bilateral & 8 & $3(27.3 \%)$ & $5(10.2 \%)$ & \\
\hline Unilateral & 52 & $8(72.7 \%)$ & $44(89.8 \%)$ & \\
\hline Interval, median (month) & $27(0,140)$ & $8(0,42)$ & $29(1,140)$ & 0.044 \\
\hline T stage & & & & 0.345 \\
\hline $3-4$ & 9 & $3(27.3 \%)$ & $6(12.2 \%)$ & \\
\hline $1-2$ & 51 & $8(72.7 \%)$ & $43(87.8 \%)$ & \\
\hline N stage & & & & 1.000 \\
\hline 123 & 24 & $4(36.4 \%)$ & $20(40.8 \%)$ & \\
\hline 0 & 36 & $7(63.6 \%)$ & $29(59.2 \%)$ & \\
\hline M stage & & & & 0.003 \\
\hline 1 & 8 & $5(45.5 \%)$ & $3(6.1 \%)$ & \\
\hline 0 & 52 & $6(54.5 \%)$ & $46(93.9 \%)$ & \\
\hline
\end{tabular}

Note-Numbers in parentheses are ranges or percentage. $p$-value based on Mann-Whitney test/Fisher Exact. 


\subsection{Malignant Ovarian Lesions}

The 11 malignant ovarian lesions comprised primary ovarian epithelial cancer $(n=6)$, moderately differentiated Sertoli-Leydig cell tumor $(n=1)$, and metastasis from breast cancer $(\mathrm{n}=4)$. Ovarian metastases from breast cancer originated from invasive ductal carcinoma $(n=2)$, lobular carcinoma $(n=1$, Figure $2 a, b)$ and angiosarcoma $(n=1)$. Their clinicopathological features are detailed in Table 2.
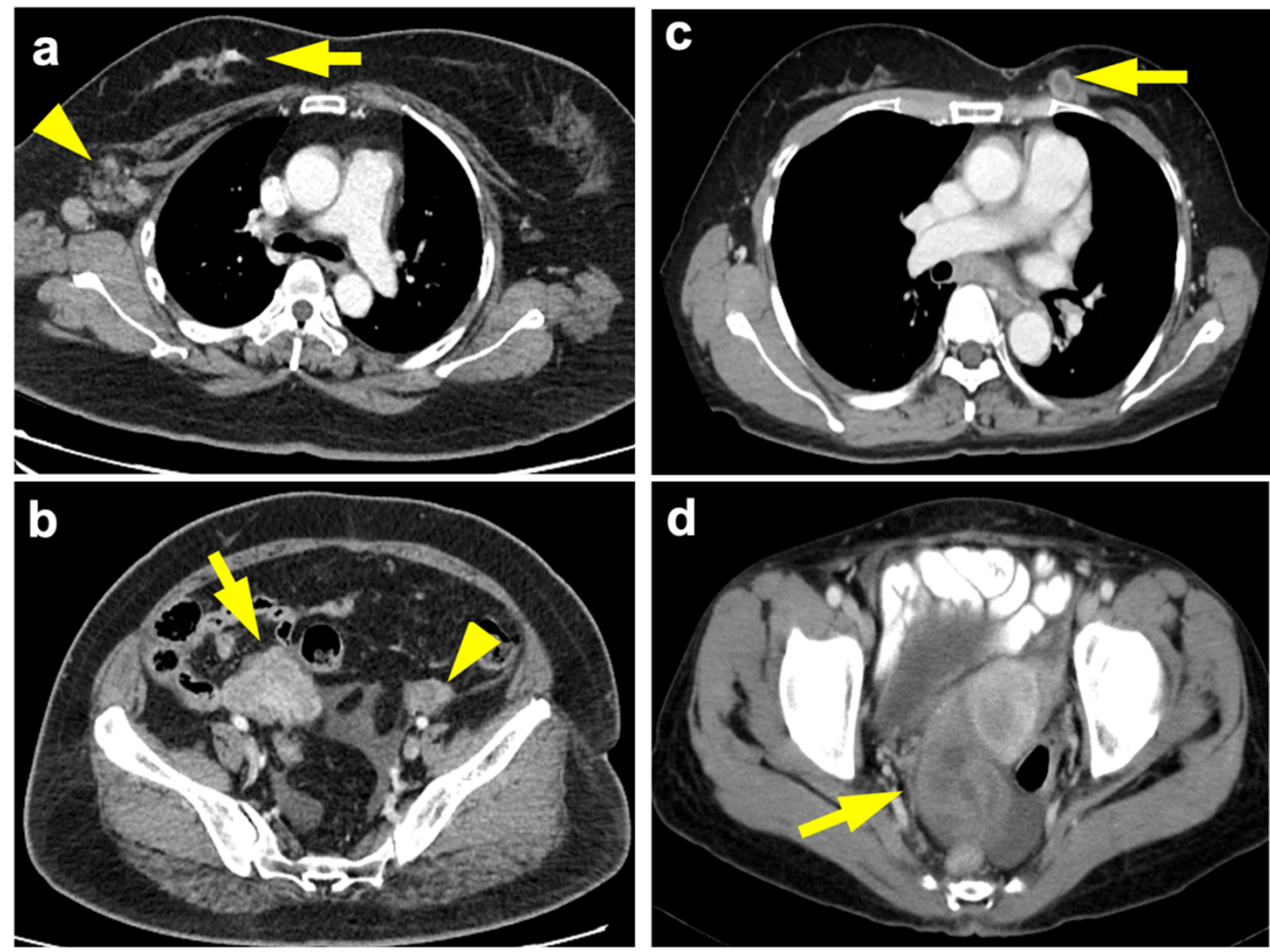

Figure 2. (a) 54-year-old woman, breast invasive lobular carcinoma (T3N2aM1) with ovarian metastasis. Contrast-enhanced axial chest CT shows infiltrative tumor at the upper outer and upper inner quadrant of the right breast (arrow) with right axillary nodal metastases (arrowhead). (b) Contrastenhanced axial pelvic CT done at the same time showed a $5.9-\mathrm{cm}$ solid tumor involving the right ovary (arrow) and a normal left ovary (arrowhead), based on final pathology. Immunohistochemistry of the breast tumor exhibited ER+, PR+ and HER2-. The levels of CA125, CA15-3, CA19-9 and CEA at documented metastasis were $38.4 \mathrm{U} / \mathrm{mL}, 15.4 \mathrm{U} / \mathrm{mL}, 21.4 \mathrm{U} / \mathrm{mL}$, and $0.84 \mathrm{ng} / \mathrm{mL}$, respectively. (c) 55-year-old woman, ovarian serous adenocarcinoma (T3cN1M1) with breast metastasis. Contrastenhanced axial chest CT shows necrotic tumors at upper inner quadrant of the left breast (arrow). (d) Contrast-enhanced axial pelvic CT 27 months ago showed complex cystic tumors (right $6.0 \mathrm{~cm}$, left $2.2 \mathrm{~cm}$ ) involving the ovaries (arrow). Immunohistochemistry of the breast tumor exhibited ER-, PR+, HER2+. The levels of CA125, CA15-3, CA19-9, and CEA at documented metastasis were 906.7 U/mL, $161.8 \mathrm{U} / \mathrm{mL}, 65.18 \mathrm{U} / \mathrm{mL}, 1.3 \mathrm{ng} / \mathrm{mL}$, respectively. Recurrence was found eight months after diagnosis of breast metastasis and patient died in 33 months. 
Table 2. Clinicopathological features of the malignant ovarian lesions.

\begin{tabular}{|c|c|c|c|c|c|c|c|c|c|c|c|c|c|c|}
\hline ID & Origin & Breast & Age & TNM & $\begin{array}{c}\text { Interval } \\
\text { (m) }\end{array}$ & Ovary & Modality & Feature * & Lat $^{+}$ & Only & CA125 & $\begin{array}{c}\text { CA15- } \\
3\end{array}$ & $\begin{array}{c}\text { CA19- } \\
9\end{array}$ & CEA \\
\hline 1 & Breast & IDC & 48 & T4N1M1 & 6 & mIDC & CT & $S$ & B & $\mathrm{N}$ & + & + & - & + \\
\hline 2 & Breast & IDC & 55 & T2NM1 & 0 & mIDC & CT & $\mathrm{S}$ & $\mathrm{U}$ & Y & - & - & + & - \\
\hline 3 & Breast & ILC & 54 & T3N2aM1 & 1 & mILC & CT & S & $\mathrm{U}$ & $\mathrm{N}$ & + & - & - & - \\
\hline 4 & Breast & AS & 40 & T2N0M1 & 32 & mAS & US & S & B & $\mathrm{N}$ & - & - & $\mathrm{N} / \mathrm{A}$ & - \\
\hline 5 & Ovary & IDC & 58 & T1cN0M0 & 42 & $\mathrm{SC}$ & US & SC & B & $\mathrm{Y}$ & + & - & - & - \\
\hline 6 & Ovary & IDC & 44 & T2NOM0 & 8 & SC & US & SC & $\mathrm{U}$ & $\mathrm{Y}$ & - & - & - & - \\
\hline 7 & Ovary & IDC & 51 & T1cN3aM0 & 34 & CCC & US & SC & $\mathrm{U}$ & $\mathrm{N}$ & - & - & $\mathrm{N} / \mathrm{A}$ & - \\
\hline 8 & Ovary & IDC & 32 & T2N0MO & 10 & CCC & CT & SC & $\mathrm{U}$ & $\mathrm{Y}$ & - & - & $\mathrm{N} / \mathrm{A}$ & - \\
\hline 9 & Ovary & IDC & 55 & T1cN2aM0 & 0 & $\mathrm{SC}$ & CT & SC & $\mathrm{U}$ & $\mathrm{Y}$ & + & + & - & $\mathrm{N} / \mathrm{A}$ \\
\hline 10 & Ovary & IDC & 60 & T1N0M0 & 1 & SC & CT & SC & $\mathrm{U}$ & $\mathrm{Y}$ & - & - & - & - \\
\hline 11 & Ovary & IDC & 38 & T4N3M1 & 41 & SL & $\mathrm{CT}$ & $\mathrm{S}$ & $\mathrm{U}$ & $\mathrm{N}$ & $\mathrm{N} / \mathrm{A}$ & N/A & $\mathrm{N} / \mathrm{A}$ & $\mathrm{N} / \mathrm{A}$ \\
\hline
\end{tabular}

Note-AS, angiosarcoma; CCC, clear cell carcinoma; IDC, invasive ductal carcinoma; ILC, invasive lobular carcinoma; MC, mucinous carcinoma; SC, serous carcinoma; UC, undifferentiated carcinoma; mIDC, metastatic invasive ductal carcinoma; mAS, metastatic angiosarcoma; mILC, metastatic lobular carcinoma; CT, computed tomography; US, ultrasonography; + , of ovary malignancy. CT features: S, solid; SC, solid-cystic; Lat $^{\dagger}$, laterality for ovarian lesions: B; bilateral; $\mathrm{U}$, unilateral. Only, ovary as the only extra-mammary solid organ metastasis. + , tumor markers elevated; - , tumor makers within normal range; N/A, non-available. TNM stages for breast cancer; Angiosarcoma staging based on staging for soft tissue sarcoma of the trunk and extremities. *, metastasis from breast cancer vs primary ovarian cancer, $p<0.05$

Ovarian lesions were detected as the first extra-mammary presentations for all the 11 patients, with one breast cancer metastasis and five primary ovarian malignancies being the only extra-mammary solid organ metastasis. Four of them were symptomatic (pain, dysmenorrhea, urinary frequency), and the pelvic ultrasound findings prompted the WBCT examination. The other seven patients were asymptomatic, with the ovarian lesions identified incidentally on routine WBCT examination. Primary ovarian cancer $(n=7)$ occurred more commonly than breast cancer with ovarian metastasis $(n=4)$, with a ratio of 1.75 . No statistically significant differences were found between the ovarian primary versus metastasis in terms of the sizes of ovarian tumors (median [range], 8.4 $\mathrm{cm}[4.9-25.2]$ vs. $8.6 \mathrm{~cm}[4.7-13.7], p=1.000$ ) or the sizes of their original breast tumors (2.2 $\mathrm{cm}[1.2-7.4]$ vs. $5.1 \mathrm{~cm}$ [1.5-9.3], $p=0.130)$. Primary ovarian cancer tended to show mixed solid-cystic, whereas the breast cancer with ovarian metastasis showed a solid appearance $(p=0.015)$ excepting the primary ovarian Sertoli-Leydig cell tumor being solid. Bilateral ovarian lesions were found in two out of the four patients with ovarian metastasis $(50 \%)$. The bilaterality of the ovarian lesions, however, did not demonstrate a statistically significant difference between primary ovarian cancers and metastasis from breast cancer. The interval of ovarian metastasis was shorter than the primary ovarian cancer (median four vs. 10 months), albeit not statistically significant. No statistical significance was observed between the ovarian metastasis and primary ovarian cancer patients in terms of their serum levels of CA 125, CA 15-3, CA19-9, and CEA. Notably, the elevation of CEA or CA19-9 or distant metastasis to the bone was only observed in breast cancer with ovarian metastasis but not in primary ovarian malignancies. The tissue expressions of estrogen receptor (ER), progesterone receptor (PR), Her2 = human epidermal growth factor receptor 2 (HER2), were found in all three breast cancers with ovarian metastases.

\subsection{Diagnostic Performance}

The diagnostic accuracy, sensitivity, and specificity, and areas under the receiver operating characteristic curve of WBCT were $98.3 \%, 100.0 \%, 98.0 \%$, and 0.99 (malignant vs. benign); $90.0 \%, 100.0 \%, 85.7 \%$, and 0.93 (metastasis vs. primary ovarian cancer), respectively. The cross-tabulation of the results is detailed in Table 3 . The solid feature had a significantly higher area under the receiver operating characteristic curve, as compared with the bilaterality feature $(0.99$ vs. $0.59, p<0.0001)$ or the combined feature $(0.99$ vs. 0.64 , $p<0.0001)$. The only false positive solid lesion was a Sertoli-Leydig tumor. 
Table 3. Diagnostic accuracy of whole-body computed tomography for ovarian lesions.

\begin{tabular}{cccccccccc}
\hline & FN & TP & TN & FP & Accuracy & Sensitivity & Specificity & PPV \\
\hline \multicolumn{7}{c}{ Malignant vs. Benign } \\
\hline Solid/SC & 0 & 11 & 48 & 1 & $98.3(91.1-100.0)$ & $100.0(71.5-100.0)$ & $98.0(89.1-99.9)$ & $91.7(61.5-99.8)$ & $100.0(92.6-100.0)$ \\
Bilateral & 8 & 3 & 44 & 5 & $78.3(65.8-87.9)$ & $27.3(6.0-61.0)$ & $89.8(77.8-96.6)$ & $37.5(8.5-75.5)$ & $84.6(71.9-93.1)$ \\
Combined & 8 & 3 & 49 & 0 & $86.7(75.4-94.1)$ & $27.3(6.0-61.0)$ & $100.0(92.7-100.0)$ & $100.0(29.2-100.0)$ & $86.0(74.2-93.7)$ \\
\hline \multicolumn{7}{c}{ Metastasis vs. Primary } \\
\hline Solid & 0 & 4 & 6 & 1 & $90.9(58.7-99.8)$ & $100.0(39.8-100.0)$ & $85.7(42.1-99.6)$ & $80.0(28.4-99.5)$ & $100.0(54.1-100.0)$ \\
Bilateral & 2 & 2 & 6 & 1 & $72.7(39.0-94.0)$ & $50.0(6.8-93.2)$ & $85.7(42.1-99.6)$ & $66.7(9.4-99.2)$ & $75.0(34.9-96.8)$ \\
Combined & 2 & 2 & 7 & 0 & $81.8(48.2-97.7)$ & $50.0(6.8-93.2)$ & $100.0(59.0-100.0)$ & $100.0(15.8-100.0)$ & $77.8(40.0-97.2)$ \\
\hline
\end{tabular}

Note-Data are numbers. In parentheses are $95 \%$ confidence intervals. SC, solid-cystic. AUC $=$ areas under the receiver operating characteristics curve, $\mathrm{TP}=$ true positive, $\mathrm{TN}=$ true negative, $\mathrm{FP}=$ false positive, $\mathrm{FN}=$ false negative.

\subsection{Breast-Ovarian Mutual Metastasis}

We identified an interesting radiological phenotype of breast primary with ovarian metastasis $(n=4)$ or ovarian primary with breast metastasis $(n=3$, Figure $2 c, d)$, defined as the mutual metastasis group in this study, as opposed to patients with double primary malignancies $(n=7)$. Mutual metastasis patients exhibited more solid rather than solid-cystic ovarian lesions $(p=0.029)$, and more distant metastatic lesions $(p=0.010)$, as compared with double primary cancer patients. Their age, diagnostic interval, as well as tissue expression of ER, PR, and HER2 were not statistically significant.

\section{Discussion}

We found that cystic ovarian lesions identified on WBCT during the breast cancer follow-up were more common to be benign, while solid-cystic lesions were likely to be primary ovarian cancers, and solid lesions possibly to be ovarian metastasis. Metastases to the ovaries from breast cancer were reported to be usually bilateral $[2,15,16]$. We found that the solid feature, either uni- or bilateral, was the most indicative for ovarian metastasis based on the histopathological evidence in the present study. Through the subgroup analysis, we identified an interesting subset phenotype of ovarian cancer, i.e., breast cancer metastasizing to ovaries or ovarian cancer metastasizing to breasts, that exhibited predominantly solid ovarian tumors, as opposed to the solid-cystic pattern in patients with double primary cancers originating from both breasts and ovaries. The present study suggested WBCT may not only detect malignant ovarian lesions but also differentiate breast metastases from primary ovarian malignancies.

Clinicopathologic characteristics of primary breast cancer provides the initial information to select patients having an increased risk of ovarian metastasis. Lobular carcinoma and invasive ductal carcinoma are the dominant pathological types with ovarian metastasis in our study. Invasive ductal carcinoma makes up the majority of cases, as shown in our cohort. Lobular carcinoma comprises only $4-15 \%$ of all malignant neoplasms of the breast $[4,7,15,17-19]$ but has three times greater metastatic tendency occurring in ovaries $[17,18]$. About $19 \%$ of lobular carcinoma with distant metastasis also have lesions in the ovary or uterus [19]. Therefore, in a patient with a history of lobular carcinoma, a new malignant ovarian mass could be more suspicious for metastatic disease. The differential diagnosis includes sex cord-stromal tumors, lymphoma/leukemia, and desmoplastic small round cell tumor of the ovary [20]. In line with our study, the majority of breast cancers with ovarian metastases were reported to have tissue expression of ER, PR, and HER2 [21]. It has been reported that breast cancer metastasis to the ovaries usually occurs in the younger, premenopausal population [7,22]; however, in our cohort, patients' age did not differ, and was mainly at perimenopausal age, as supported by other studies $[4,21]$. The time interval between the diagnosis of initial breast cancer with succeeding primary ovarian cancer was shorter than with ovarian metastasis [23]. 
Understanding the incidence is helpful in this clinical scenario. The probability of ovarian metastasis in breast cancer patients is estimated to be $6.7 \%(4 / 60)$ in the present study, and ovarian metastases are found mostly in women with advanced stages of breast cancer. Based on the present study, a newly found adnexal mass in a woman with prior breast cancer is more commonly benign. The benign and malignant disease resulted in a 4.5:1 ratio, which is in line with two independent cohort studies with 129 patients [5] and 54 patients [24], respectively. Within the scope of malignant masses, we demonstrated that the primary ovarian cancer and metastatic disease resulted in a 1.75:1 ratio. This is supported by another study with 27 patients diagnosed with primary epithelial ovarian cancer and nine had metastatic disease, resulting in a 3:1 ratio [4].

The finding of ovarian metastases in the ovaries when the disease has the systemic character requires systemic treatment and pelvic/abdominal surgery to remove the adnexal mass is not always required. On the other hand, even the incidental finding of a small solid-cystic or purely solid adnexal mass on the WBCT or pelvic transvaginal sonography in a woman with a history of breast cancer makes an early differential diagnosis extremely important. Our result is supported by the literature, showing even small primary tumors may result in metastatic disease to the ovaries [21,24]. Metastases to the ovaries from breast cancer can be relatively small solid masses; in nearly half of cases the size of the ovary is not enlarged $[15,25]$. From hematogenous spread, the ovarian metastases from breast cancer are mainly nested in the ovarian medulla and/or cortex [26,27]. In contrast, primary ovarian cancers are more commonly located in the ovarian surface epithelium and superficial cortex, accompanied by fallopian tube involvement [28]. In line with our study, the transvaginal ultrasonography features of ovarian metastases appear to be solid if involved by lymphoma or metastases from the stomach, breast and uterus, whereas being multicystic with irregular borders if derived from the colon, rectum or biliary tract [10-12]. Unilateral tumors appear more often in the primary ovarian cancer group and bilateral disease in the ovarian metastasis $[4,13]$. Our study showed the laterality added little value to select breast cancer metastasis in the present cohort.

The breast-ovarian mutual metastasis patients also demonstrated more extensive distant metastasis at the time of ovarian diagnosis. Other poor prognostic factors of breast cancer, such as co-existent other metastatic sites [3], stage III-IV [24,29], and large positive lymph nodes [19], are positively correlated with metastatic breast cancer to the ovaries. Although $B R C A$ mutations increase the risk of ovarian cancer, the incidence of ovarian metastasis did not differ between the $B R C A$ mutation carriers and noncarrier breast cancer patients [30]. It has been reported that $75 \%$ of patients were asymptomatic, and $42 \%$ of patients exhibited advanced-stage pelvic extent or extra-abdominal metastases [7], highlighting the importance of WBCT in detecting distant metastasis. A study based on PET-CT also supported that breast cancer with solid ovarian metastasis with multiple metastases included omentum, liver, and bone [31], whereas synchronous double primaries demonstrated bilateral adnexal cystic tumors with omentum and peritoneal spread [31]. Because both cancer and the physiological change of the ovary might demonstrate avid glucose uptake, the morphological features from the CT part of PET-CT might also aid the differential diagnosis in this regard.

\section{Limitation}

Some limitations warrant notification while interpreting our data. First, this is a singlecenter retrospective study. Due to the limited number of patients with malignant cases, subgroup analysis of metastasis type and imaging features of malignant ovaries could not be carried out simultaneously. That could introduce bias to the data, and the sample size was not sufficient to uncover modest differences. To prevent selection bias and uncontrolled confounding factors, such as non-surgical chemotherapy regimens, we did not intend to investigate the impact on prognosis, but focused on the utility of WBCT in the clinical decisions pathway. Nevertheless, to our knowledge, the present study includes the largest number of ovarian cancers for WBCT analysis. Second, the case number in the ovarian 
malignancy group might be underestimated, because our study defined the ground truth to be histopathological evidence only. The prevalence of perimenopausal breast cancer patients with ovarian metastasis might lead the readers to think that bilateral oophorectomy might be beneficial even when the contralateral ovary appears to be normal [7]. The number of cases with malignant ovarian lesions in the present report is very small, so one must be very cautious with the interpretation of the results. The current results translate to select the surgical candidate and improve survival, but might warrant a larger series to confirm.

\section{Conclusions}

In conclusion, cystic ovarian lesions identified on WBCT during the breast cancer follow-up are more common to be benign, while solid-cystic lesions are likely to be primary ovarian cancers, and solid lesions possibly to be ovarian metastasis. WBCT may help diagnose incidental ovarian tumors on WBCT in patients with prior breast cancer and guide the disease management.

Author Contributions: Conceptualization, S.-H.N., C.-H.L., A.C. and G.L.; Investigation, P.-C.H., Y.-H.J., H.-Y.H. and Y.-T.H.; Supervision, S.-H.N. and C.-H.L.; Validation, R.-C.W. and Y.-C.L.; Writing-original draft, P.-C.H., H.-Y.H., Y.-T.H.; Writing-review \& editing, G.L. All authors have read and agreed to the published version of the manuscript.

Funding: This work was supported by Chang Gung Medical Foundation grant CLRPG3K0022, CLRPG3K0023, Ministry of Science and Technology (Taiwan) MOST 110-2628-B-182A-018.

Institutional Review Board Statement: This retrospective research was approved by Chang Gung Medical Foundation Institutional Review Board (IRB) No. 202101049B0.

Informed Consent Statement: This retrospective study was conducted in accordance with the guidelines of the Declaration of Helsinki and approved by the Ethics Committee of the Chang Gung Memorial Hospital. The need for written informed consent was waived by the Ethics Committee.

Data Availability Statement: All data generated or analyzed during this study are included in this published article.

Acknowledgments: The authors thank Hsin-Ying Lu for data analysis, Kuan-Ying Lu for document preparation, and all the members of the Cancer Center, Chang Gung Memorial Hospital.

Conflicts of Interest: The authors declare that they have no conflicts of interest in this study.

\section{References}

1. Siegel, R.L.; Miller, K.D.; Fuchs, H.E.; Jemal, A. Cancer Statistics, 2021. CA Cancer J. Clin. 2021, 71, 7-33. [CrossRef] [PubMed]

2. Tian, W.; Zhou, Y.; Wu, M.; Yao, Y.; Deng, Y. Ovarian metastasis from breast cancer: A comprehensive review. Clin. Transl. Oncol. 2019, 21, 819-827. [CrossRef] [PubMed]

3. Peters, I.T.; van Zwet, E.W.; Smit, V.T.; Liefers, G.J.; Kuppen, P.J.; Hilders, C.G.; Trimbos, J.B. Prevalence and Risk Factors of Ovarian Metastases in Breast Cancer Patients $<41$ Years of Age in the Netherlands: A Nationwide Retrospective Cohort Study. PLoS ONE 2017, 12, e0168277. [CrossRef]

4. Tserkezoglou, A.; Kontou, S.; Hadjieleftheriou, G.; Apostolikas, N.; Vassilomanolakis, M.; Sikiotis, K.; Salamalekis, E.; Tseke, P.; Magiakos, G. Primary and metastatic ovarian cancer in patients with prior breast carcinoma. Pre-operative markers and treatment results. Anticancer Res. 2006, 26, 2339-2344. [PubMed]

5. Simpkins, F.; Zahurak, M.; Armstrong, D.; Grumbine, F.; Bristow, R. Ovarian malignancy in breast cancer patients with an adnexal mass. Obstet. Gynecol. 2005, 105, 507-513. [CrossRef] [PubMed]

6. $\quad$ Bergfeldt, K.; Rydh, B.; Granath, F.; Gronberg, H.; Thalib, L.; Adami, H.O.; Hall, P. Risk of ovarian cancer in breast-cancer patients with a family history of breast or ovarian cancer: A population-based cohort study. Lancet 2002, 360, 891-894. [CrossRef]

7. Bigorie, V.; Morice, P.; Duvillard, P.; Antoine, M.; Cortez, A.; Flejou, J.F.; Uzan, S.; Darai, E.; Barranger, E. Ovarian metastases from breast cancer: Report of 29 cases. Cancer 2010, 116, 799-804. [CrossRef] [PubMed]

8. Runowicz, C.D.; Leach, C.R.; Henry, N.L.; Henry, K.S.; Mackey, H.T.; Cowens-Alvarado, R.L.; Cannady, R.S.; Pratt-Chapman, M.L.; Edge, S.B.; Jacobs, L.A.; et al. American Cancer Society/American Society of Clinical Oncology Breast Cancer Survivorship Care Guideline. CA Cancer J. Clin. 2016, 66, 43-73. [CrossRef] [PubMed]

9. Gradishar, W.J.; Moran, M.S.; Abraham, J.; Aft, R.; Agnese, D.; Allison, K.H.; Blair, S.L.; Burstein, H.J.; Dang, C.; Elias, A.D.; et al. NCCN Guidelines Insights: Breast Cancer, Version 4. 2021: Featured Updates to the NCCN Guidelines. J. Natl. Compr. Cancer Netw. 2021, 19, 484-493. [CrossRef] [PubMed] 
10. Antila, R.; Jalkanen, J.; Heikinheimo, O. Comparison of secondary and primary ovarian malignancies reveals differences in their pre- and perioperative characteristics. Gynecol. Oncol. 2006, 101, 97-101. [CrossRef]

11. Guerriero, S.; Alcazar, J.L.; Pascual, M.A.; Ajossa, S.; Olartecoechea, B.; Hereter, L. Preoperative diagnosis of metastatic ovarian cancer is related to origin of primary tumor. Ultrasound Obstet. Gynecol. 2012, 39, 581-586. [CrossRef] [PubMed]

12. Testa, A.C.; Ferrandina, G.; Timmerman, D.; Savelli, L.; Ludovisi, M.; Van Holsbeke, C.; Malaggese, M.; Scambia, G.; Valentin, L. Imaging in gynecological disease (1): Ultrasound features of metastases in the ovaries differ depending on the origin of the primary tumor. Ultrasound Obstet. Gynecol. 2007, 29, 505-511. [CrossRef] [PubMed]

13. Bruls, J.; Simons, M.; Overbeek, L.I.; Bulten, J.; Massuger, L.F.; Nagtegaal, I.D. A national population-based study provides insight in the origin of malignancies metastatic to the ovary. Virchows Arch. 2015, 467, 79-86. [CrossRef] [PubMed]

14. Cimino-Mathews, A. Novel uses of immunohistochemistry in breast pathology: Interpretation and pitfalls. Mod. Pathol. 2021, 34, 62-77. [CrossRef]

15. Chuang, A.Y.; Watkins, J.C.; Young, R.H.; Lerwill, M.F. Lobular Carcinoma of the Breast Metastatic to the Ovary: A Clinicopathologic Study of 38 Cases. Am. J. Surg Pathol. 2022, 46, 179-189. [CrossRef] [PubMed]

16. Karaosmanoglu, A.D.; Onur, M.R.; Salman, M.C.; Usubutun, A.; Karcaaltincaba, M.; Ozmen, M.N.; Akata, D. Imaging in secondary tumors of the ovary. Abdom. Radiol. 2019, 44, 1493-1505. [CrossRef] [PubMed]

17. Ferlicot, S.; Vincent-Salomon, A.; Medioni, J.; Genin, P.; Rosty, C.; Sigal-Zafrani, B.; Freneaux, P.; Jouve, M.; Thiery, J.P.; SastreGarau, X. Wide metastatic spreading in infiltrating lobular carcinoma of the breast. Eur. J. Cancer 2004, 40, 336-341. [CrossRef] [PubMed]

18. Weigelt, B.; Peterse, J.L.; van't Veer, L.J. Breast cancer metastasis: Markers and models. Nat. Rev. Cancer 2005, 5, 591-602. [CrossRef]

19. Pimentel, C.; Becquet, M.; Lavoue, V.; Henno, S.; Leveque, J.; Ouldamer, L. Ovarian Metastases from Breast Cancer: A Series of 28 Cases. Anticancer Res. 2016, 36, 4195-4200.

20. Skotnicki, P.; Sas-Korczynska, B.; Wohadlo, L.; Jakubowicz, J.; Blecharz, P.; Reinfuss, M.; Walasek, T. Distant metastases from invasive lobular breast carcinoma classic type-Treatment and prognosis. Eur. J. Gynaecol. Oncol. 2016, 37, 666-670. [CrossRef]

21. Cerkauskaite, D.; Zilinskas, K.; Varnelis, P.; Oreibi, M.E.; Asejev, V.; Dulskas, A. Ovarian metastases from breast cancer: A report of 24 cases. J. Gynecol. Obstet. Hum. Reprod. 2021, 50, 102075. [CrossRef] [PubMed]

22. Moore, E.K.; Roylance, R.; Rosenthal, A.N. Breast cancer metastasising to the pelvis and abdomen: What the gynaecologist needs to know. BJOG Int. J. Obstet. Gynaecol. 2012, 119, 788-794. [CrossRef] [PubMed]

23. Olawaiye, A.; Caesar, L.; Walsh, D.; Lyman, M.; Yeh, J.; Rodabaugh, K.; Marchetti, D.; Lele, S.; Odunsi, K. Analysis of the time interval between diagnoses in women with double primary breast and ovarian or primary peritoneal cancers. Gynecol. Oncol. 2004, 94, 796-802. [CrossRef] [PubMed]

24. Hann, L.E.; Lui, D.M.; Shi, W.; Bach, A.M.; Selland, D.L.; Castiel, M. Adnexal masses in women with breast cancer: US findings with clinical and histopathologic correlation. Radiology 2000, 216, 242-247. [CrossRef]

25. de Waal, Y.R.; Thomas, C.M.; Oei, A.L.; Sweep, F.C.; Massuger, L.F. Secondary ovarian malignancies: Frequency, origin, and characteristics. Int. J. Gynecol. Cancer 2009, 19, 1160-1165. [CrossRef]

26. Moore, R.G.; Chung, M.; Granai, C.O.; Gajewski, W.; Steinhoff, M.M. Incidence of metastasis to the ovaries from nongenital tract primary tumors. Gynecol. Oncol. 2004, 93, 87-91. [CrossRef]

27. Peters, I.T.; van der Steen, M.A.; Huisman, B.W.; Hilders, C.G.; Smit, V.T.; Vahrmeijer, A.L.; Sier, C.F.; Trimbos, J.B.; Kuppen, P.J Morphological and phenotypical features of ovarian metastases in breast cancer patients. BMC Cancer 2017, 17, 206. [CrossRef]

28. Kondi-Pafiti, A.; Kairi-Vasilatou, E.; Iavazzo, C.; Dastamani, C.; Bakalianou, K.; Liapis, A.; Hassiakos, D.; Fotiou, S. Metastatic neoplasms of the ovaries: A clinicopathological study of 97 cases. Arch. Gynecol. Obstet. 2011, 284, 1283-1288. [CrossRef]

29. Abd El hafez, A.; Monir, A. Diagnostic spectrum of ovarian masses in women with breast cancer; magnetic resonance imaging: Histopathology correlation. Ann. Diagn. Pathol. 2013, 17, 441-447. [CrossRef]

30. Song, Y.; Barry, W.T.; Seah, D.S.; Tung, N.M.; Garber, J.E.; Lin, N.U. Patterns of recurrence and metastasis in BRCA1/BRCA2associated breast cancers. Cancer 2020, 126, 271-280. [CrossRef]

31. Yadav, B.S.; Sharma, S.C.; Robin, T.P.; Sams, S.; Elias, A.D.; Kaklamani, V.; Kelly Marcom, P.; Schaefer, S.; Morris, G.J. Synchronous primary carcinoma of breast and ovary versus ovarian metastases. Semin. Oncol. 2015, 42, e13-e24. [CrossRef] [PubMed] 